hep-th/9503132

submitted to:

Journal of Mathematical Physics

\title{
Covariant algebraic method for calculation of the low-energy heat kernel
}

\author{
I. G. Avramidi a) b) c) \\ Department of Mathematics, University of Greifswald \\ Jahnstr. 15a, 17489 Greifswald, Germany
}

\begin{abstract}
Using our recently proposed covariant algebraic approach the heat kernel for a Laplace-like differential operator in low-energy approximation is studied. Neglecting all the covariant derivatives of the gauge field strength (Yang-Mills curvature) and the covariant derivatives of the potential term of third order and higher a closed formula for the heat kernel as well as its diagonal is obtained. Explicit formulas for the coefficients of the asymptotic expansion of the heat kernel diagonal in terms of the Yang-Mills curvature, the potential term and its first two covariant derivatives are obtained.
\end{abstract}

\footnotetext{
a) Alexander von Humboldt Fellow

b) On leave of absence from the Research Institute for Physics, Rostov State University, Stachki 194, Rostov-on-Don 344104, Russia

c) E-mail: avramidi@math-inf.uni-greifswald.d400.de
} 


\section{INTRODUCTION}

The heat kernel, the kernel of the one-parameter semigroup (or the heat operator), $U(t)=\exp (-t H)$, for an elliptic differential operator $H$ acting on a section of a vector bundle over a manifold $M$, plays a very important role in various areas of mathematical physics, especially in quantum field theory and quantum gravity. ${ }^{1-28}$ It determines among others such fundamental objects of the quantum field theory as the Green function, the kernel of the resolvent ${ }^{2}$

$$
G(\lambda)=(H+\lambda)^{-1}=\int_{0}^{\infty} d t e^{-t \lambda} U(t),
$$

the zeta-function,(see Ref. 15),

$$
\zeta(p)=\operatorname{Tr} H^{-p}=\frac{1}{\Gamma(p)} \int_{0}^{\infty} d t t^{p-1} \operatorname{Tr} U(t),
$$

where $\operatorname{Tr}$ is the functional trace, the functional determinant, Det $H$, and, hence, the oneloop effective action

$$
\Gamma_{(1)}=\frac{1}{2} \log \operatorname{Det} H=-\frac{1}{2} \zeta^{\prime}(0) .
$$

The most important operators in quantum field theory are the second order elliptic operators of the form

$$
H=-\square+Q,
$$

where $\square=g^{\mu \nu} \nabla_{\mu} \nabla_{\nu}, \nabla_{\mu}$ is a connection on the vector bundle and $Q$ is an endomorphism of this bundle. In other words the operator $H$ acts on a multiplet of quantum fields, $\phi(x)$, $Q(x)$ is a matrix valued potential term, $\nabla_{\mu}=\partial_{\mu}+\mathcal{A}_{\mu}, \mathcal{A}_{\mu}$ is a gauge (Yang-Mills) field, the gauge field strength (Yang-Mills curvature), $\mathcal{R}_{\mu \nu}$, being given by the commutator of covariant derivatives

$$
\left[\nabla_{\mu}, \nabla_{\nu}\right] \phi=\mathcal{R}_{\mu \nu} \phi .
$$

$M$ is taken to be a $d$-dimensional Riemannian manifold $M$ with a metric, $g_{\mu \nu}$, of Euclidean (positive) signature. 
Obviously, the heat kernel is calculable exactly only in exceptional cases of background fields configurations, (see, for example Ref. 14). That is why for studying the general case there is a need to develop approximate methods of calculations. Two such approximation schemes are available: ${ }^{3}$ i) the high-energy one, which corresponds to weak rapidly varying background fields (short waves), and ii) the low-energy approximation corresponding to the strong slowly varying background fields (long waves). The high-energy approximation was studied in Refs. 16-19 where the heat kernel and the effective action in second ${ }^{16,17}$ and third ${ }^{18,19}$ order in background fields (curvatures) were calculated. The low-energy approximation in various settings was studied in Refs. 20-22. The authors of these papers summed up some particular terms in the heat kernel asymptotic expansion, such as the scalar curvature terms ${ }^{20,21}$ or terms without derivatives of the potential term ${ }^{22}$ etc.

In our recent papers ${ }^{23-28}$ the status of the low-energy approximation in quantum gravity and gauge theories was analyzed. We developed a new purely algebraic covariant approach for calculating the heat kernel near diagonal. The point is that in low-energy approximation the covariant derivatives of the curvatures and the potential term (but not the curvature and the potential term themselves!) are small. Therefore, one can treat them perturbatively, the zeroth order of this perturbation theory corresponding to the covariantly constant background fields.

To gain greater insight into how the low-energy heat kernel looks like, one can take into account a finite number of low-order covariant derivatives of the background fields, and neglect all the covariant derivatives of higher orders. Then there exist a set of differential operators, actually approximate Killing vectors, that together with the background fields and their low-order derivatives generate a finite dimensional Lie algebra. If one does not neglect the higher derivatives of the background fields then this algebra is infinitedimensional. This procedure is very similar to the polynomial approximation of functions of real variables. The difference is that we are dealing, in general, with the covariant derivatives and the curvatures.

In the previous papers we considered the case of covariantly constant gauge field strength and the potential term in flat space, $\nabla_{\mu} \mathcal{R}_{\alpha \beta}=\nabla_{\mu} Q=R_{\alpha \beta \gamma \delta}=0,{ }^{23-25}$ and the case of covariantly constant Riemann curvature and the potential term without the 
Yang-Mills curvature, $\nabla_{\mu} R_{\alpha \beta \gamma \delta}=\nabla_{\mu} Q=\mathcal{R}_{\mu \nu}=0,{ }^{26,27}$. In the Ref. 28 this method was applied for the investigation of the effective potential of a non-Abelian gauge theory.

In this paper we are going to take into account the next terms in the low-energy approximation in the flat space, i.e. we consider the case $\nabla_{\mu} \mathcal{R}_{\alpha \beta}=\nabla_{\mu} \nabla_{\nu} \nabla_{\lambda} Q=0$, $R_{\alpha \beta \gamma \delta}=0$, and take into account the first and the second derivatives of the potential term in the presence of the covariantly constant Yang-Mills curvature.

\section{COVARIANT ALGEBRAIC APPROACH}

\section{A. Low-energy Lie algebra}

As we will study only local effects in the low-energy approximation, we will not take care about the topology of the manifold $M$. To be precise one can take, for example, $\mathbb{R}^{\mathrm{d}}$. We assume only that in some region of the manifold the background fields satisfy approximately the following low-energy local conditions

$$
R_{\mu \nu \alpha \beta}=0, \quad \nabla_{\alpha} \mathcal{R}_{\mu \nu}=0, \quad \nabla_{\mu} \nabla_{\nu} \nabla_{\lambda} Q=0
$$

Commuting the covariant derivatives here leads to the following restrictions

$$
\left[\mathcal{R}_{\alpha \beta}, \mathcal{R}_{\mu \nu}\right]=0, \quad\left[\mathcal{R}_{\alpha \beta}, Q_{; \nu}\right]=0, \quad\left[\mathcal{R}_{\alpha \beta}, Q_{; \mu \nu}\right]=0, \quad\left[\mathcal{R}_{\alpha \beta},\left[\mathcal{R}_{\mu \nu}, Q\right]\right]=0
$$

where $Q_{; \mu} \equiv \nabla_{\mu} Q, Q_{; \nu \mu} \equiv \nabla_{\mu} \nabla_{\nu} Q$. Although the conditions (2.2) do not imply directly

$$
\left[\mathcal{R}_{\alpha \beta}, Q\right]=0
$$

we assume (2.3) to be true since this follows from (2.2) if we suppose that (2.2) should be valid for arbitrary gauge group. Therefore, we assume, actually, the background to be approximately Abelian, i.e. all the nonvanishing background quantities, $\mathcal{R}_{\alpha \beta}, Q, Q_{; \mu}, Q_{; \nu \mu}$, are assumed to commute with each other, in other words, to lie in the Cartan subalgebra of the algebra of the gauge group, so that in addition to (2.2) and (2.3) it holds

$$
\left[Q, Q_{; \mu}\right]=0, \quad\left[Q, Q_{; \mu \nu}\right]=0, \quad\left[Q_{; \mu}, Q_{; \alpha \beta}\right]=0
$$


For our purposes, it is helpful to introduce the following parametrization of the potential term

$$
Q=M-\beta^{i k} L_{i} L_{k},
$$

where $(i=1, \ldots, p), p \leq d, \beta^{i k}$ is some constant symmetric nondegenerate $p \times p$ matrix, $M$ is a covariantly constant matrix and $L_{i}$ are some matrices with vanishing second covariant derivative

$$
\nabla_{\mu} M=0, \quad \nabla_{\mu} \nabla_{\nu} L_{i}=0 .
$$

The derivatives of the potential term take then the form

$$
Q_{; \mu}=-2 E_{\mu i} \beta^{i k} L_{k}, \quad Q_{; \nu \mu}=2 P_{\nu \mu}
$$

where

$$
E_{\mu i}=\nabla_{\mu} L_{i}, \quad P_{\mu \nu}=-E_{\mu k} \beta^{k i} E_{\nu i} .
$$

Thus we have a nilpotent Lie algebra, $\left\{\nabla_{\mu}, \mathcal{R}_{\alpha \beta}, M, L_{i}, E_{\mu i}\right\}$, with following nontrivial commutators

$$
\left[\nabla_{\mu}, \nabla_{\nu}\right]=\mathcal{R}_{\mu \nu}, \quad\left[\nabla_{\mu}, L_{i}\right]=E_{\mu i},
$$

and the center $\left\{\mathcal{R}_{\alpha \beta}, M, L_{i}, E_{\mu i}\right\}$. Introducing the generators $X_{A}=\left(\nabla_{\mu}, L_{i}\right),(A=$ $1, \ldots, D), D=d+p$, one can rewrite these commutation relations in a more compact form

$$
\begin{gathered}
{\left[X_{A}, X_{B}\right]=\mathcal{F}_{A B},} \\
{\left[X_{A}, \mathcal{F}_{C D}\right]=\left[\mathcal{F}_{A B}, \mathcal{F}_{C D}\right]=0,}
\end{gathered}
$$

where $\mathcal{F}_{A B}$ is a matrix

$$
\left(\mathcal{F}_{A B}\right)=\left(\begin{array}{cc}
\mathcal{R}_{\mu \nu} & E_{\mu i} \\
-E_{\nu k} & 0
\end{array}\right),
$$

that we call the generalized curvature. The operator $H$ (1.4) can now be written in the form

$$
H=-\gamma^{A B} X_{A} X_{B}+M,
$$

where

$$
\left(\gamma^{A B}\right)=\left(\begin{array}{cc}
g^{\mu \nu} & 0 \\
0 & \beta^{i k}
\end{array}\right) .
$$


The matrices $\beta^{i k}$ and $\gamma^{A B}$ play the role of metrics and can be used to raise and to lower the small and the capital Latin indices respectively.

\section{B. Analytic functions of the generalized curvature}

Let us now introduce the following matrix notation for the generalized curvature

$$
\begin{gathered}
\mathcal{F}=\left(\mathcal{F}_{B}^{A}\right)=\left(\begin{array}{cc}
\mathcal{R} & E \\
-\bar{E} & 0
\end{array}\right), \\
\mathcal{R}=\left(\mathcal{R}^{\mu}{ }_{\nu}\right), \quad E=\left(E^{\mu}{ }_{i}\right), \quad \bar{E}=\left(\bar{E}^{k}{ }_{\nu}\right)=\left(E_{\nu}{ }^{k}\right),
\end{gathered}
$$

and consider analytic functions of this matrix.

The powers of the matrix $\mathcal{F}(2.14)$ have the following general structure

$$
\mathcal{F}^{0}=1, \quad \mathcal{F}^{k}=\left(\begin{array}{cc}
J_{k} & J_{k-1} E \\
-\bar{E} J_{k-1} & -\bar{E} J_{k-2} E
\end{array}\right), \quad k \geq 1,
$$

where the $d \times d$ matrices $J_{k}=\left(J_{k}{ }^{\mu}{ }_{\nu}\right)$ are defined by the recursion relations

$$
J_{k+1}=\mathcal{R} J_{k}+P J_{k-1},
$$

with the following initial conditions

$$
J_{-1}=0, \quad J_{0}=1 .
$$

The low-order matrices are

$$
\begin{aligned}
& J_{0}=1, \\
& J_{1}=\mathcal{R}, \\
& J_{2}=\mathcal{R}^{2}+P, \\
& J_{3}=\mathcal{R}^{3}+\mathcal{R} P+P \mathcal{R}, \\
& J_{4}=\mathcal{R}^{4}+\mathcal{R}^{2} P+\mathcal{R} P \mathcal{R}+P \mathcal{R}^{2}+P^{2},
\end{aligned}
$$

etc. It is clear that $J_{k}$ is a polynomial of two noncommuting 'variables' $\mathcal{R}$ and $P$ with all possible terms of the dimension $\mathcal{R}^{k}$ with coefficients +1 , the total number, $N_{k}$, of terms being

$$
N_{k}=\sum_{0 \leq n \leq[k / 2]}\left(\begin{array}{c}
k-n \\
n
\end{array}\right)
$$


The polynomials $J_{k}$ can be calculated with the help of the generating function

$$
F(z)=\left(1-z \mathcal{R}-z^{2} P\right)^{-1}=\sum_{k \geq 0} z^{k} J_{k}
$$

in the following way

$$
J_{k}=\left.\frac{1}{k !} \frac{\partial^{k}}{\partial z^{k}} F(z)\right|_{z=0}=\oint_{C} \frac{d z}{2 \pi i} z^{-k-1} F(z),
$$

where the integral is taken along a sufficiently small closed contour $C$ that encircles the origin counter-clockwise, so that $F(z)$ is analytic inside this contour.

Using the symmetry properties of the matrices $\mathcal{R}$ and $P$

$$
\mathcal{R}^{T}=-g \mathcal{R} g^{-1}, \quad P^{T}=g P g^{-1},
$$

where $g=\left(g_{\mu \nu}\right)$, we find

$$
J_{2 k}^{T}=g J_{2 k} g^{-1}, \quad J_{2 k+1}^{T}=-g J_{2 k+1} g^{-1},
$$

and

$$
\left(\mathcal{F}^{2 k}\right)^{T}=\gamma \mathcal{F}^{2 k} \gamma^{-1}, \quad\left(\mathcal{F}^{2 k+1}\right)^{T}=-\gamma \mathcal{F}^{2 k+1} \gamma^{-1},
$$

where $\gamma=\left(\gamma_{A B}\right)$. Therefore, the traces of odd order polynomials as well as the traces of odd powers of $\mathcal{F}$ vanish

$$
\operatorname{tr} J_{2 k+1}=0, \quad \operatorname{tr} \mathcal{F}^{2 k+1}=0 .
$$

Hence using (2.15) we obtain the traces of even powers

$$
\operatorname{tr} \mathcal{F}^{2 k}=\operatorname{tr}\left(J_{2 k}+P J_{2 k-2}\right) .
$$

Thus any analytic function of the matrix $\mathcal{F}$ has the form

$$
\begin{aligned}
f(\mathcal{F}) & =\sum_{n \geq 0} \frac{1}{n !} f^{(n)} \mathcal{F}^{n}=\left(\begin{array}{cc}
U & V E \\
-\bar{E} V & f(0)-\bar{E} W E
\end{array}\right) \\
& =\oint_{C} \frac{d z}{2 \pi i} \frac{1}{z} f\left(z^{-1}\right)(1-z \mathcal{F})^{-1}
\end{aligned}
$$


where $U=\left(U_{\nu}^{\mu}\right)$ and $W=\left(W_{\nu}^{\mu}\right)$ are symmetric and $V=\left(V_{\nu}^{\mu}\right)$ antisymetric $d \times d$ matrices

$$
U^{T}=g U g^{-1}, \quad W^{T}=g W g^{-1}, \quad V^{T}=-g V g^{-1}
$$

defined by

$$
\begin{aligned}
U & =\sum_{n \geq 0} \frac{1}{n !} f^{(n)} J_{n}=\oint_{C} \frac{d z}{2 \pi i} \frac{1}{z} f\left(z^{-1}\right) F(z), \\
V & =\sum_{n \geq 1} \frac{1}{n !} f^{(n)} J_{n-1}=\oint_{C} \frac{d z}{2 \pi i} f\left(z^{-1}\right) F(z), \\
W & =\sum_{n \geq 2} \frac{1}{n !} f^{(n)} J_{n-2}=\oint_{C} \frac{d z}{2 \pi i} z f\left(z^{-1}\right) F(z) .
\end{aligned}
$$

Herefrom we find the trace of an analytic function, $f(\mathcal{F})(2.27)$,

$$
\begin{aligned}
\operatorname{tr} f(\mathcal{F}) & =\operatorname{tr}(U+P W)=\operatorname{tr} f(0)+\sum_{n \geq 2} \frac{1}{n !} f^{(n)} \operatorname{tr}\left(J_{n}+P J_{n-2}\right) \\
& =\oint_{C} \frac{d z}{2 \pi i} f\left(z^{-1}\right) \operatorname{tr}\left\{\left(z^{-1}+z P\right) F(z)\right\}
\end{aligned}
$$

and the determinant

$$
\operatorname{det}\left(\frac{f(\mathcal{F})}{f(0)}\right)=\exp \left\{\oint_{C} \frac{d z}{2 \pi i} \log \left(\frac{f\left(z^{-1}\right)}{f(0)}\right) \operatorname{tr}\left\{\left(z^{-1}+z P\right) F(z)\right\}\right\} .
$$

These formulas take especially simple form in a particular case when the matrices $\mathcal{R}$ and $P$ commute,

$$
[\mathcal{R}, P]=0
$$

In this case the generating function $F(z)(2.20)$ can be evaluated exactly

$$
F(z)=\frac{1}{\Delta}\left(\frac{R_{+}}{1-z R_{+}}-\frac{R_{-}}{1-z R_{-}}\right),
$$

where

$$
R_{ \pm}=\frac{1}{2}(\mathcal{R} \pm \Delta), \quad \Delta=\sqrt{\mathcal{R}^{2}+4 P}
$$

Thereby we find

$$
J_{k}=\frac{1}{\Delta}\left(R_{+}^{k+1}-R_{-}^{k+1}\right) .
$$


Using these polynoms we obtain from (2.29)-(2.31)

$$
\begin{aligned}
U & =\Delta^{-1}\left(R_{+} f\left(R_{+}\right)-R_{-} f\left(R_{-}\right)\right) \\
& =\frac{1}{2}\left(f\left(R_{+}\right)+f\left(R_{-}\right)\right)+\frac{1}{2} \mathcal{R} V, \\
V & =\Delta^{-1}\left(f\left(R_{+}\right)-f\left(R_{-}\right)\right), \\
W & =\Delta^{-1}\left(R_{+}^{-1} f\left(R_{+}\right)-R_{-}^{-1} f\left(R_{-}\right)\right),
\end{aligned}
$$

and, consequently, from (2.32) and (2.33)

$$
\begin{gathered}
\operatorname{tr} f(\mathcal{F})=\operatorname{tr}\left(f\left(R_{+}\right)+f\left(R_{-}\right)\right) . \\
\operatorname{det}\left(\frac{f(\mathcal{F})}{f(0)}\right)=\operatorname{det}\left(\frac{f\left(R_{+}\right)}{f(0)} \frac{f\left(R_{-}\right)}{f(0)}\right) .
\end{gathered}
$$

\section{The heat operator}

Now using the algebra (2.10) we are able to calculate the heat kernel operator, $\exp (-t H)$. For an algebra of this kind in Refs. 23,24 it was proven a theorem that gives the heat operator in terms of an integral over the corresponding Lie group. Namely,

$$
\begin{aligned}
\exp (-t H)= & (4 \pi t)^{-D / 2} \operatorname{det}\left(\frac{\sinh (t \mathcal{F})}{t \mathcal{F}}\right)^{-1 / 2} \exp (-t M) \\
& \times \int_{\mathbb{R}^{\mathrm{D}}} d k \gamma^{1 / 2} \exp \left\{-\frac{1}{4 t} k_{A}(t \mathcal{F} \operatorname{coth}(t \mathcal{F}))_{B}^{A} k^{B}+k^{A} X_{A}\right\},
\end{aligned}
$$

where $\gamma=\operatorname{det} \gamma_{A B},\left(\gamma_{A B}\right)=\left(\gamma^{A B}\right)^{-1}$.

Thus we have expressed the heat kernel operator in terms of the operator $\exp \left(k^{A} X_{A}\right)$. Splitting the integration variables $\left(k^{A}\right)=\left(q^{\mu}, \omega^{i}\right)$ in (2.43) and using the CampbellHausdorf formula we single out the noncommutative part

$$
\begin{aligned}
\exp \left(k^{A} X_{A}\right)=\exp \left(q^{\mu} \nabla_{\mu}+\omega^{i} L_{i}\right) & =\exp \left(\omega^{i} L_{i}\right) \exp \left(\frac{1}{2}\left[q^{\mu} \nabla_{\mu}, \omega^{i} L_{i}\right]\right) \exp \left(q^{\mu} \nabla_{\mu}\right) \\
& =\exp \left\{\omega^{i}\left(L_{i}+\frac{1}{2} q^{\mu} E_{\mu i}\right)\right\} \exp \left(q^{\mu} \nabla_{\mu}\right) .
\end{aligned}
$$

Further, we employ the parametrization

$$
t \mathcal{F} \operatorname{coth}(t \mathcal{F})=\left(\begin{array}{cc}
B(t) & t A(t) E \\
-t \bar{E} A(t) & 1-t^{2} \bar{E} C(t) E
\end{array}\right),
$$


and integrate over $\omega$ in $(2.43)$ to yield

$$
\begin{aligned}
\exp (-t H)= & (4 \pi t)^{-d / 2} \operatorname{det}\left(\frac{\sinh (t \mathcal{F})}{t \mathcal{F}}\right)^{-1 / 2} \operatorname{det} Z(t)^{1 / 2} \exp \left\{-t M+t L_{i} Z^{i}{ }_{k}(t) L^{k}\right\} \\
& \times \int_{\mathbb{R}^{\mathrm{d}}} d q g^{1 / 2} \exp \left\{-\frac{1}{4 t} q^{\mu} D_{\mu \nu}(t) q^{\nu}+t L_{i} Y_{\mu}^{i}(t) q^{\mu}\right\} \exp \left(q^{\mu} \nabla_{\mu}\right),
\end{aligned}
$$

where

$$
\begin{gathered}
Z(t)=\left(Z_{k}^{i}(t)\right)=\left(1-t^{2} \bar{E} C(t) E\right)^{-1}, \\
Y(t)=\left(Y_{\mu}^{i}(t)\right)=Z(t) \bar{E}(1+A(t)), \\
D(t)=\left(D_{\nu}^{\mu}(t)\right)=B(t)-t^{2}(1-A(t)) E Z(t) \bar{E}(1+A(t)) .
\end{gathered}
$$

Further, using (2.5) and (2.7) it is not difficult to prove

$$
\begin{gathered}
L_{i} Y_{\nu}^{i}(t)=-\frac{1}{2} Q_{; \mu}\left(1+t^{2} C P\right)_{\nu}^{-1 \mu} \\
L_{i} Z_{k}^{i}(t) L^{k}=M-Q+\frac{1}{4} t^{2} Q_{; \mu}\left[\left(1+t^{2} C P\right)^{-1} C\right]_{\nu}^{\mu} Q^{; \nu} \\
\operatorname{det} Z(t)=\operatorname{det}\left(1+t^{2} C P\right)^{-1},
\end{gathered}
$$

where $P=\left(P_{\nu}^{\mu}\right)=-E \bar{E}$ is given by (2.8). Substituting these expressions in (2.46) we arrive finally to the heat operator in the form

$$
\begin{aligned}
& \exp (-t H)=(4 \pi t)^{-d / 2} \exp \left\{-t Q+\Phi(t)+\frac{1}{4} t^{3} Q_{; \mu} \Psi^{\mu \nu}(t) Q_{; \nu}\right\} \\
& \quad \times \int_{\mathbb{R}^{\mathrm{d}}} d q g^{1 / 2} \exp \left\{-\frac{1}{4 t} q^{\mu} D_{\mu \nu}(t) q^{\nu}-\frac{t}{2} Q_{; \mu}\left(\delta_{\nu}^{\mu}+A_{\nu}^{\mu}(t)\right) q^{\nu}\right\} \exp \left(q^{\mu} \nabla_{\mu}\right),
\end{aligned}
$$

where

$$
\begin{gathered}
D(t)=\left(D_{\nu}^{\mu}(t)\right)=B(t)+t^{2}(1-A(t)) P\left(1+t^{2} C(t) P\right)^{-1}(1+A(t)) \\
\Phi(t)=-\frac{1}{2} \log \operatorname{det}\left(\frac{\sinh (t \mathcal{F})}{t \mathcal{F}}\right)-\frac{1}{2} \log \operatorname{det}\left(1+t^{2} C(t) P\right) \\
\Psi(t)=\left(\Psi_{\nu}^{\mu}(t)\right)=\left(1+t^{2} C(t) P\right)^{-1} C(t)
\end{gathered}
$$


the matrices $A(t), B(t)$ and $C(t)$ being defined from (2.29)-(2.31) and (2.45) by

$$
\begin{aligned}
& B(t)=\oint_{C} \frac{d z}{2 \pi i} \frac{t}{z^{2}} \operatorname{coth}\left(t z^{-1}\right) F(z), \\
& A(t)=\oint_{C} \frac{d z}{2 \pi i} \frac{t}{z} \operatorname{coth}\left(t z^{-1}\right) F(z), \\
& C(t)=\oint_{C} \frac{d z}{2 \pi i} t \operatorname{coth}\left(t z^{-1}\right) F(z),
\end{aligned}
$$

with $F(z)$ given by $(2.20)$. It is worth noting that in the parametrization

$$
\frac{\sinh (t \mathcal{F})}{t \mathcal{F}}=\left(\begin{array}{cc}
K(t) & t S(t) E \\
-t \bar{E} S(t) & 1-t^{2} \bar{E} N(t) E
\end{array}\right)
$$

where

$$
\begin{aligned}
& K(t)=\oint_{C} \frac{d z}{2 \pi i} \frac{t}{z^{2}} \sinh \left(t z^{-1}\right) F(z), \\
& S(t)=\oint_{C} \frac{d z}{2 \pi i} \frac{t}{z} \sinh \left(t z^{-1}\right) F(z), \\
& N(t)=\oint_{C} \frac{d z}{2 \pi i} t \sinh \left(t z^{-1}\right) F(z),
\end{aligned}
$$

one can calculate the determinant of this $D \times D$ matrix as follows

$$
\begin{aligned}
\operatorname{det}\left(\frac{\sinh (t \mathcal{F})}{t \mathcal{F}}\right) & =\operatorname{det} K \operatorname{det}\left[1+t^{2}\left(N-S K^{-1} S\right) P\right] \\
& =\operatorname{det}\left(1+t^{2} N P\right) \operatorname{det}\left[K-t^{2} S P\left(1+t^{2} N P\right)^{-1} S\right] .
\end{aligned}
$$

\section{THE HEAT KERNEL}

\section{A. Closed formula for the heat kernel diagonal}

To obtain the heat kernel in coordinate representation we have just to act with the heat operator, $\exp (-t H)$, on the coordinate $\delta$-function

$$
U\left(t \mid x, x^{\prime}\right)=\exp (-t H) \mathcal{P}\left(x, x^{\prime}\right) g^{-1 / 2} \delta\left(x-x^{\prime}\right)
$$


where $\mathcal{P}\left(x, x^{\prime}\right)$ is the parallel displacement operator (matrix) of the field $\phi$ from the point $x^{\prime}$ to the point $x$ along the geodesic. It is not difficult to show ${ }^{23,24}$ that

$$
\exp \left(q^{\mu} \nabla_{\mu}^{x}\right) \mathcal{P}\left(x, x^{\prime}\right) g^{-1 / 2} \delta\left(x-x^{\prime}\right)=\mathcal{P}\left(x, x^{\prime}\right) g^{-1 / 2} \delta\left(x-x^{\prime}+q\right)
$$

Hence the integration over $q$ in (2.53) becomes trivial and we obtain the heat kernel

$$
\begin{aligned}
& U\left(t \mid x, x^{\prime}\right)=(4 \pi t)^{-d / 2} \mathcal{P}\left(x, x^{\prime}\right) \exp \left\{-t Q(x)+\Phi(t)+\frac{1}{4} t^{3} Q_{; \mu}(x) \Psi^{\mu \nu}(t) Q_{; \nu}(x)\right\} \\
& \quad \times \exp \left\{-\frac{1}{4 t}\left(x-x^{\prime}\right)^{\mu} D_{\mu \nu}(t)\left(x-x^{\prime}\right)^{\nu}+\frac{t}{2} Q_{; \mu}(x)\left(\delta_{\nu}^{\mu}+A_{\nu}^{\mu}(t)\right)\left(x-x^{\prime}\right)^{\nu}\right\} .
\end{aligned}
$$

Expanding this expression in a power series in $\left(x-x^{\prime}\right)$ one can easily get all the coincidence limits of covariant derivatives of the heat kernel. In particular, the heat kernel diagonal has a very simple form

$$
[U(t)]=U(t \mid x, x)=(4 \pi t)^{-d / 2} \exp \left\{-t Q+\Phi(t)+\frac{1}{4} t^{3} Q_{; \mu} \Psi^{\mu \nu}(t) Q_{; \nu}\right\}
$$

This is the main result of this paper. The formula (3.4) exhibits the general structure of the heat kernel diagonal. Namely, one sees immediately how the potential term and its first derivatives enter the result. The complete nontrivial information is contained only in a scalar, $\Phi(t)$, and a tensor, $\Psi_{\mu \nu}(t)$, functions which are constructed purely from the Yang-Mills curvature $\mathcal{R}_{\mu \nu}$ and the second derivatives of the potential term, $\nabla_{\mu} \nabla_{\nu} Q$. So we conclude that the coefficients of the heat kernel asymptotic expansion are constructed from three different types of scalar (connected) blocks, $Q, \Phi_{(n)}(\mathcal{R}, \nabla \nabla Q)$ and $\nabla_{\mu} Q \Psi_{(n)}^{\mu \nu}(\mathcal{R}, \nabla \nabla Q) \nabla_{\nu} Q$. We will calculate the coefficients of the heat kernel asymptotic expansion explicitly in the Subsects. III.B and III.C.

Before we do this let us consider the particular case (2.34) when the matrices $\mathcal{R}$ and $P$ commute, i.e.

$$
\mathcal{R}_{\nu}^{\mu} P_{\alpha}^{\nu}=P_{\nu}^{\mu} \mathcal{R}_{\alpha}^{\nu}
$$

Making use of the formulas (2.45) and (2.36)-(2.42) we get

$$
\begin{gathered}
\operatorname{det}\left(\frac{\sinh (t \mathcal{F})}{t \mathcal{F}}\right)=\operatorname{det}\left(\frac{\sinh \left(t R_{+}\right)}{t R_{+}} \frac{\sinh \left(t R_{-}\right)}{t R_{-}}\right), \\
C(t)=-\frac{1}{t^{2} P}-\frac{\sinh (t \Delta)}{t \Delta} \frac{1}{\sinh \left(t R_{+}\right) \sinh \left(t R_{-}\right)} .
\end{gathered}
$$


Now from (2.55) and (2.56) it follows

$$
\begin{gathered}
\Phi(t)=-\frac{1}{2} \log \operatorname{det}\left(\frac{\sinh (t \Delta)}{t \Delta}\right), \\
\Psi(t)=\frac{1}{t^{2} P}\left[\frac{\Delta}{2 t P} \frac{\cosh (t \mathcal{R})-\cosh (t \Delta)}{\sinh (t \Delta)}+1\right] .
\end{gathered}
$$

Thus in this special case the heat kernel diagonal reads

$$
\begin{aligned}
{[U(t)] } & =(4 \pi t)^{-d / 2} \operatorname{det}\left(\frac{\sinh (t \Delta)}{t \Delta}\right)^{-1 / 2} \\
& \times \exp \left\{-t Q+\frac{1}{4} t Q_{; \mu}\left[\frac{1}{P}\left(\frac{\Delta}{2 t P} \frac{\cosh (t \mathcal{R})-\cosh (t \Delta)}{\sinh (t \Delta)}+1\right)\right]_{\nu}^{\mu} Q^{; \nu}\right\} .
\end{aligned}
$$

If the second derivatives of the potential vanish, $P_{\mu \nu}=\frac{1}{2} \nabla_{\mu} \nabla_{\nu} Q=0$, then we have from (3.8)-(3.10)

$$
\begin{aligned}
& \Phi(t)=-\frac{1}{2} \log \operatorname{det}\left(\frac{\sinh (t \mathcal{R})}{t \mathcal{R}}\right), \\
& \Psi(t)=\frac{1}{t^{2} \mathcal{R}^{2}}(t \mathcal{R} \operatorname{coth}(t \mathcal{R})-1) .
\end{aligned}
$$

The heat kernel diagonal is now

$$
\begin{aligned}
{[U(t)]=} & (4 \pi t)^{-d / 2} \operatorname{det}\left(\frac{\sinh (t \mathcal{R})}{t \mathcal{R}}\right)^{-1 / 2} \\
& \times \exp \left\{-t Q+\frac{1}{4} t Q_{; \mu}\left(\frac{1}{\mathcal{R}^{2}}(t \mathcal{R} \operatorname{coth}(t \mathcal{R})-1)\right)_{\nu}^{\mu} Q^{; \nu}\right\} .
\end{aligned}
$$

This is the first order correction to the case of covariantly constant potential ${ }^{23}$ when additionally the first derivatives of the potential are taken into account.

In the case of vanishing Yang-Mills curvature, $\mathcal{R}=0$, we have similarly

$$
\begin{gathered}
\Phi(t)=-\frac{1}{2} \log \operatorname{det}\left(\frac{\sinh (2 t \sqrt{P})}{2 t \sqrt{P}}\right), \\
\Psi(t)=-\frac{1}{(t \sqrt{P})^{3}}(\tanh (t \sqrt{P})-t \sqrt{P}),
\end{gathered}
$$

and the heat kernel diagonal has the form

$$
\begin{aligned}
{[U(t)]=} & (4 \pi t)^{-d / 2} \operatorname{det}\left(\frac{\sinh (2 t \sqrt{P})}{2 t \sqrt{P}}\right)^{-1 / 2} \\
& \times \exp \left\{-t Q-\frac{1}{4} Q_{; \mu}\left(\frac{\tanh (t \sqrt{P})-t \sqrt{P}}{P^{3 / 2}}\right)_{\nu}^{\mu} Q^{; \nu}\right\}
\end{aligned}
$$


This determines the low-energy approximation without the gauge fields. The formulas (3.10), (3.13) and (3.16) can be used, in particular, to check the results for the coefficients of the heat kernel asymptotic expansion obtained in general case.

\section{B. Asymptotic expansion of the heat kernel diagonal}

Let us now calculate the Taylor expansions of the functions $\Phi(t)(2.55)$ and $\Psi(t)$ (2.56). Using the well known series ${ }^{29}$

$$
\log \frac{\sinh x}{x}=\sum_{n \geq 1} \frac{2^{2 n-1} B_{2 n}}{n(2 n) !} x^{2 n}
$$

where $B_{n}$ are the Bernulli numbers ${ }^{29}$, we obtain from (2.32)

$$
\log \operatorname{det}\left(\frac{\sinh (t \mathcal{F})}{t \mathcal{F}}\right)=\sum_{n \geq 1} \frac{2^{2 n-1} B_{2 n}}{n(2 n) !} t^{2 n} \operatorname{tr}\left(J_{2 n}+P J_{2 n-2}\right)
$$

Further, using another series ${ }^{29}$

$$
x \operatorname{coth} x=\sum_{n \geq 0} \frac{2^{2 n} B_{2 n}}{(2 n) !} x^{2 n}
$$

we find from (2.57)-(2.59) the functions $B(t), A(t)$ and $C(t)$

$$
\begin{gathered}
B(t)=\sum_{n \geq 0} \frac{2^{2 n} B_{2 n}}{(2 n) !} t^{2 n} J_{2 n}, \\
A(t)=\sum_{n \geq 1} \frac{2^{2 n} B_{2 n}}{(2 n) !} t^{2 n-1} J_{2 n-1}, \\
C(t)=\sum_{n \geq 1} \frac{2^{2 n} B_{2 n}}{(2 n) !} t^{2 n-2} J_{2 n-2} .
\end{gathered}
$$

Now it is not difficult to calculate

$$
\begin{aligned}
\log \operatorname{det}\left(1+t^{2} C(t) P\right) & =\sum_{k \geq 1} t^{2 k} 2^{2 k-1} \sum_{1 \leq N \leq k} \frac{(-1)^{N}}{N} \sum_{\substack{1 \leq n_{1}, \ldots, n_{N} \leq k \\
n_{1}+\cdots+n_{N}=k}} \frac{B_{2 n_{1}}}{\left(2 n_{1}\right) !} \cdots \frac{B_{2 n_{N}}}{\left(2 n_{N}\right) !} \\
& \times \operatorname{tr}\left(P J_{2 n_{1}-2} \cdots P J_{2 n_{N}-2}\right) .
\end{aligned}
$$


Thereby combining (3.18) and (3.23) we find the function $\Phi(t)$

$$
\Phi(t)=\sum_{k \geq 1} \frac{(-1)^{k}}{(2 k) !} t^{2 k} \Phi_{2 k}
$$

where

$$
\begin{aligned}
& \Phi_{2 k}=\operatorname{tr}\left\{\frac{2^{2 k-2}}{k}\left|B_{2 k}\right|\left(\mathcal{R} J_{2 k-1}+2(k+1) P J_{2 k-2}\right)\right. \\
& \left.+2^{2 k-1} \sum_{2 \leq N \leq k} \frac{1}{N} \sum_{\substack{1 \leq n_{1}, \ldots, n_{N} \leq k-1 \\
n_{1}+\cdots+n_{N}=k}} \frac{(2 k) !}{\left(2 n_{1}\right) ! \cdots\left(2 n_{N}\right) !}\left|B_{2 n_{1}}\right| \cdots\left|B_{2 n_{N}}\right| P J_{2 n_{1}-2} \cdots P J_{2 n_{N}-2}\right\} .
\end{aligned}
$$

Here we used the recursion relations (2.16) and the property of the Bernulli numbers $B_{2 k}=(-1)^{k+1}\left|B_{2 k}\right|,(k \geq 1)$. Since all the polynoms $J_{k}$ have only positive coefficients we see that $\Phi_{2 k}$ are also polynoms of the matrix $\mathcal{R}$ and $P$ with positive coefficients.

Similarly, from (3.22) and (2.56) we obtain the expansion of the matrix $\Psi$

$$
\Psi(t)=\sum_{k \geq 0} \frac{(-1)^{k}}{(2 k+3) !} t^{2 k} \Psi_{2 k}
$$

where

$$
\begin{aligned}
\Psi_{2 k}= & (2 k+3) 2^{2 k+2}\left|B_{2 k+2}\right| J_{2 k} \\
& +2^{2 k+2} \sum_{2 \leq N \leq k+1 ;} \sum_{\substack{1 \leq n_{1}, \ldots, n_{N} \leq k \\
n_{1}+\cdots+n_{N}=k+1}} \frac{(2 k+3) !}{\left(2 n_{1}\right) ! \cdots\left(2 n_{N}\right) !}\left|B_{2 n_{1}}\right| \cdots\left|B_{2 n_{N}}\right| \\
& \times J_{2 n_{1}-2} P J_{2 n_{2}-2} P \cdots P J_{2 n_{N}-2} .
\end{aligned}
$$

Evidently, $\Psi_{2 k}$ also is a polynom of $\mathcal{R}$ and $P$ having only positive coefficients. Analogously, using the explicit formulas (3.20)-(3.22) one can calculate the Taylor series of the matrix $D(2.54)$ too. We will not list here the result because it is not needed for the calculation of the heat kernel diagonal.

Finally, substituting the series (3.24) and (3.26) in (3.4) we obtain the expansion of the heat kernel diagonal

$$
[U(t)]=(4 \pi t)^{-d / 2} \exp \left(\sum_{k \geq 1}(-1)^{[(k+1) / 2]} \frac{t^{k}}{k !} b_{k}\right)
$$


where the coefficients $b_{k}$ are defined by

$$
b_{1}=Q, \quad b_{2 k}=\Phi_{2 k}, \quad b_{2 k+1}=\frac{1}{4} Q_{; \mu} \Psi_{2 k-2}^{\mu \nu} Q_{; \nu}
$$

and all have only positive coefficients. The coefficients of the usual asymptotic expansion of the heat kernel diagonal, ${ }^{6}$

$$
[U(t)]=(4 \pi t)^{-d / 2} \sum_{k \geq 0} \frac{(-t)^{k}}{k !} a_{k}
$$

are given then by

$$
a_{k}=\sum_{1 \leq N \leq k ;} \sum_{\substack{1 \leq k_{1} \leq \ldots \leq k_{N} \leq k \\ k_{1}+\cdots+k_{N}=k}}(-1)^{\left[\left(3 k_{1}+1\right) / 2\right]+\cdots+\left[\left(3 k_{N}+1\right) / 2\right]} \frac{k !}{\left(k_{1} !\right)^{2} \cdots\left(k_{N} !\right)^{2}} b_{k_{1}} \cdots b_{k_{N}} .
$$

\section{Explicit results for low-order coefficients}

Using the formulas of the previous subsection it is not difficult to calculate the coefficients of the asymptotic expansion of the heat kernel diagonal explicitly. We list below some low-order terms

$$
\begin{aligned}
a_{1}= & b_{1} \\
a_{2}= & b_{1}^{2}-b_{2} \\
a_{3}= & b_{1}^{3}-3 b_{1} b_{2}-b_{3} \\
a_{4}= & b_{1}^{4}-6 b_{1}^{2} b_{2}-4 b_{1} b_{3}+3 b_{2}^{2}+b_{4} \\
a_{5}= & b_{1}^{5}-10 b_{1}^{3} b_{2}-10 b_{1}^{2} b_{3}+15 b_{1} b_{2}^{2}+5 b_{1} b_{4}+10 b_{2} b_{3}+b_{5}, \\
a_{6}= & b_{1}^{6}-15 b_{1}^{4} b_{2}-20 b_{1}^{3} b_{3}+15 b_{1}^{2} b_{4}+45 b_{1}^{2} b_{2}^{2}+60 b_{1} b_{2} b_{3}+6 b_{1} b_{5}-15 b_{2} b_{4}+10 b_{3}^{2}-b_{6}, \\
a_{7}= & b_{1}^{7}-21 b_{1}^{5} b_{2}-35 b_{1}^{4} b_{3}+35 b_{1}^{3} b_{4}+190 b_{1}^{3} b_{2}^{2}+21 b_{1}^{2} b_{5}+210 b_{1}^{2} b_{2} b_{3}-7 b_{1} b_{6} \\
& -105 b_{1} b_{2} b_{4}+70 b_{1} b_{3}^{2}-21 b_{2} b_{5}-35 b_{3} b_{4}-b_{7}, \\
a_{8}= & b_{1}^{8}-28 b_{1}^{6} b_{2}-56 b_{1}^{5} b_{3}+70 b_{1}^{4} b_{4}+210 b_{1}^{4} b_{2}^{2}+56 b_{1}^{3} b_{5}+560 b_{1}^{3} b_{2} b_{3}-28 b_{1}^{2} b_{6}-420 b_{1}^{2} b_{2} b_{4} \\
& +280 b_{1}^{2} b_{3}^{2}-8 b_{1} b_{7}-168 b_{1} b_{2} b_{5}-280 b_{1} b_{3} b_{4}+28 b_{2} b_{6}-56 b_{3} b_{5}+35 b_{4}^{2}+b_{8},
\end{aligned}
$$


where

$$
\begin{aligned}
b_{1}= & Q, \\
b_{2}= & \frac{1}{6} \operatorname{tr}\left(\mathcal{R}^{2}+4 P\right), \\
b_{4}= & \frac{1}{15} \operatorname{tr}\left(\mathcal{R}^{2}+4 P\right)^{2}, \\
b_{6}= & \frac{8}{63} \operatorname{tr}\left(\mathcal{R}^{6}+12 \mathcal{R}^{4} P+39 \mathcal{R}^{2} P^{2}+9 \mathcal{R} P \mathcal{R} P+64 P^{3}\right), \\
b_{8}= & \frac{8}{15} \operatorname{tr}\left(\mathcal{R}^{8}+16 \mathcal{R}^{6} P+\frac{153}{3} \mathcal{R}^{4} P^{2}+\frac{592}{3} \mathcal{R}^{2} P^{3}+24 \mathcal{R}^{3} P \mathcal{R} P\right. \\
& \left.+\frac{64}{3} \mathcal{R}^{2} P \mathcal{R}^{2} P+\frac{176}{3} \mathcal{R} P \mathcal{R} P^{2}+256 P^{4}\right),
\end{aligned}
$$

and

$$
\begin{aligned}
& b_{3}=\frac{1}{2} Q_{; \mu} Q^{; \mu}, \\
& b_{5}=\frac{2}{3} Q_{; \mu}\left(\mathcal{R}^{2}+6 P\right)^{\mu}{ }_{\nu} Q^{; \nu}, \\
& b_{7}=\frac{8}{3} Q_{; \mu}\left(\mathcal{R}^{4}+9 \mathcal{R}^{2} P+\mathcal{R} P \mathcal{R}+\frac{51}{2} P^{2}\right)^{\mu}{ }_{\nu} Q^{; \nu} .
\end{aligned}
$$

It should be reminded here that the matrix $P$ is composed of the second derivatives of the potential term, $P=\left(P_{\nu}^{\mu}\right), P_{\mu \nu}=\frac{1}{2} \nabla_{\mu} \nabla_{\nu} Q, \mathcal{R}=\left(\mathcal{R}^{\mu}{ }_{\nu}\right)$ is the Yang-Mills curvature and the trace 'tr' is taken only with respect to vector (Greek) indices, the gauge ones being intact. For example,

$$
\operatorname{tr} \mathcal{R}^{2}=\mathcal{R}^{\mu}{ }_{\nu} \mathcal{R}_{\mu}^{\nu}, \quad \operatorname{tr} P=P_{\mu}^{\mu}=\frac{1}{2} \square Q,
$$

etc. Notice that the term $\square Q$ appears only in combination $b_{2}=\frac{1}{6}\left(\mathcal{R}_{\mu \nu} \mathcal{R}^{\nu \mu}+2 \square Q\right)$.

\section{TRACE OF THE HEAT KERNEL}

Let us discuss now the functional trace of the heat kernel

$$
\operatorname{Tr} U(t)=\int_{M} d x g^{1 / 2} \operatorname{tr}[U(t)] .
$$

It is well known that if the manifold $M$ is compact then there is a classic (standard) asymptotic expansion as $t \rightarrow 0^{6}$

$$
\operatorname{Tr} U(t) \sim(4 \pi t)^{-d / 2} \sum_{k \geq 0} t^{k / 2} A_{k / 2}(H, M) .
$$


The coefficients $A_{k / 2}(H, M)$ are spectral invariants of the operator $H$ which are calculable in form of integrals of local invariants of background fields over the manifold (and the boundary). In case of manifolds without boundary this expansion can be obtained by direct term by term integration of the asymptotic expansion of the heat kernel diagonal (3.30) over the manifold, i.e.

$$
A_{k}=(-1)^{k} k ! \int_{M} d x g^{1 / 2} \operatorname{tr} a_{k}, \quad A_{k+1 / 2}=0 .
$$

For noncompact manifold the situation is far more complicated. The problem is that the integrals like (4.3) for noncompact manifolds do not converge, in general. This depends on the behavior of the background fields at the infinity.

Let us consider, for example, a complete noncompact manifold without boundary, $\partial M=\emptyset$. In the case when all background fields have only compact support or fall off sufficiently rapidly at the infinity the invariants $A_{k}$ are also well defined except

$$
A_{0}=\text { const } \cdot \operatorname{vol}(M) \text {. }
$$

If we introduce an auxiliary operator $H_{0}$ with the symbol $H_{0}(x, \xi)=H_{L}\left(x_{0}, \xi\right)$, where $H_{L}\left(x_{0}, \xi\right)$ is the leading symbol of the operator $H$ and $x_{0}$ is some fixed point, having trivial coefficients

$$
a_{0}\left(H_{0}\right)=1, \quad a_{k}\left(H_{0}\right)=0, \quad k \geq 1,
$$

then the trace of the difference of two heat kernels is well defined for noncompact manifolds too,

$$
\operatorname{Tr}\left(\exp (-t H)-\exp \left(-t H_{0}\right)\right) \sim(4 \pi t)^{-d / 2} \sum_{k \geq 1} t^{k} A_{k}(H, M) .
$$

This case is similar to the standard compact one.

However, if the background fields (curvature or the potential term) do not decrease at the infinity then the asymptotics of the trace of the heat kernel changes radically. In this case all the coefficients $A_{k}$ (4.3) diverge and. This means that the trace of the heat kernel has a different nonstandard asymptotics. One can show that the main term of the 
asymptotics can be always obtained by

$$
\begin{aligned}
\left.\operatorname{Tr} U(t)\right|_{t \rightarrow 0} & \left.\sim(2 \pi)^{-d} \operatorname{tr} \int_{M} d x \int_{\mathbb{R}^{\mathrm{d}}} d \xi \exp (-t H(x, \xi))\right|_{t \rightarrow 0} \\
& \left.\sim(4 \pi t)^{-d / 2} \operatorname{tr} \int_{M} d x g^{1 / 2} \exp (-t Q(x))\right|_{t \rightarrow 0},
\end{aligned}
$$

where $H(x, \xi)$ is the symbol of the operator $H(1.4)$.

Therefore, the asymptotics depends essentially on the behavior of the potential term at the infinity. If the potential is positive definite and increases at the infinity then the integral over the manifold always exist and determines the main term of the asymptotics. For example, if the potential at the infinity behaves like

$$
\left.Q(x)\right|_{x \rightarrow \infty}=P_{\mu \nu} x^{\mu} x^{\nu}+O(x)
$$

and the matrix $P=\left(P_{\nu}^{\mu}\right)$ is nondegenerate, then we obtain from (4.7) asymptotically

$$
\operatorname{Tr} U(t) \sim(2 t)^{-d} \operatorname{det} P^{-1 / 2}+O\left(t^{-d+1}\right)
$$

This is a typical example of a nonstandard asymptotics. Instead of usual behavior, $\sim t^{-d / 2}$, we have a more singular one, $\sim t^{-d}$. Generally, there can be far more complicated asymptotics, including the logarithmic terms, $(\log t)^{\alpha}$, etc., depending on the the asymptotic behavior of the potential term at the infinity.

Let us now calculate the trace of the heat kernel in our case. We assumed that the background fields satisfy the low-energy conditions (2.1)-(2.4) in some region of the manifold $M$. Let us suppose the manifold $M$ to be $\mathbb{R}^{\mathrm{d}}$ and the conditions (2.1)-(2.4) to hold everywhere. Then the formula for the heat kernel diagonal (3.4) is valid everywhere too. If we write it in the form

$$
\begin{aligned}
{[U(t)]=} & (4 \pi t)^{-d / 2} \operatorname{det}\left(\frac{\sinh (t \mathcal{F})}{t \mathcal{F}}\right)^{-1 / 2} \operatorname{det}\left(1+t^{2} C(t) P\right)^{-1 / 2} \\
& \times \exp \left\{-t M-t\left(x-x_{0}\right)^{\mu} \Pi_{\mu \nu}(t)\left(x-x_{0}\right)^{\nu}\right\}
\end{aligned}
$$

where $x_{0}$ is some fixed point and

$$
\Pi(t)=\left(\Pi_{\nu}^{\mu}(t)\right)=P\left(1+t^{2} C(t) P\right)^{-1},
$$


and suppose the matrix $P$ to be nondegenerate, then one can integrate $(4.10)$ over $\mathbb{R}^{\mathrm{d}}$ to get

$$
\operatorname{Tr} U(t)=(2 t)^{-d} \operatorname{det}\left(\frac{\sinh (t \mathcal{F})}{t \mathcal{F}}\right)^{-1 / 2} \operatorname{det} P^{-1 / 2} \exp (-t M) .
$$

This expression also has a nonclassic asymptotics, $\operatorname{Tr} U(t) \sim$ const $\cdot t^{-d}$. In particular case of commuting matrices $\mathcal{R}$ and $P$ the trace of the heat kernel takes especially simple form

$$
\operatorname{Tr} U(t)=\operatorname{det}\{2(\cosh (t \Delta)-\cosh (t \mathcal{R}))\}^{-1 / 2} \exp (-t M)
$$

which reduces to

$$
\operatorname{Tr} U(t)=\operatorname{det}(2 \sinh (t \sqrt{P}))^{-1} \exp (-t M)
$$

when $\mathcal{R}=0$.

It should be noted that the standard form of the asymptotics of the trace of the heat kernel (4.2) is the basis for the regularization and renormalization procedure in quantum field theory. ${ }^{2}$ That is why the non-standard asymptotics may cause serious technical problems in the theory of quantum fields on noncompact manifolds with background fields that do not fall off at infinity. For example, the analytical structure of the zeta function (1.2) in non-standard case will be completely different. This is the consequence of the fact that in this non-standard situation the physical quantum states are not well defined.

\section{CONCLUSION}

In present paper we continued the investigation of the heat kernel in low-energy approximation initiated in our recent papers. ${ }^{23-28}$ We developed further a manifestly covariant algebraic approach for calculation of the heat operator, i.e. the one-parameter semigroup, $\exp (-t H)$, proposed in these papers and applied it to evaluate the heat kernel and, especially, the heat kernel diagonal. We were able to take into account in the asymptotic expansion of the heat kernel diagonal all the terms that contain only the Yang-Mills curvature, $\mathcal{R}_{\mu \nu}$, the potential term $Q$ and its two low-order derivatives, $\nabla_{\mu} Q$ and $\nabla_{\mu} \nabla_{\nu} Q$.

We obtained a closed formula for the heat kernel diagonal that can be treated as a resummation of the asymptotic expansion, those terms being summed up first. The 
covariant algebraic approach employed in this paper is especially adequate and effective to study the low-energy approximation. It seems that it can be developed deeper and that it can be formulated a general technique for systematic calculation of the low-energy heat kernel, a kind of low-energy covariant perturbation theory.

\section{ACKNOWLEDGEMENTS}

I would like to thank R. Schimming for many helpful discussions and J. Eichhorn for the hospitality at the University of Greifswald. This work was supported, in part, by the Alexander von Humboldt Foundation.

1 J. S. Schwinger, Phys. Rev. 82, 664 (1951).

2 B. S. De Witt, Dynamical theory of groups and fields (Gordon and Breach, New York, 1965); Phys. Rep. C 19, 296 (1975); in General Relativity, edited by S. Hawking and W. Israel (Cambridge University Press, Cambridge, 1979).

3 G. A. Vilkovisky, in Quantum Gravity, edited by S. Christensen (Adam Hilger, Bristol, 1983), p. 169.

4 A. O. Barvinsky and G. A. Vilkovisky, Phys. Rep. C 119, 1 (1985).

${ }^{5}$ I. G. Avramidi, Nucl. Phys. B 355, 712 (1991); Phys. Lett. B 238, 92 (1990); Teor. Mat. Fiz. 79, 219 (1989).

${ }^{6}$ P. B. Gilkey, J. Diff. Geom. 10, 601 (1975); Invariance theory, the heat equation and the Atiyah - Singer index theorem (Publish or Perish, Wilmington, 1984).

7 R. Schimming, Beitr. Anal. 15, 77 (1981); Math. Nachr. 148, 145 (1990).

8 R. Schimming, in Analysis, Geometry and Groups: A Riemann Legacy Volume, edited by H. M. Srivastava and Th. M. Rassias, (Hadronic Press, Palm Harbor, 1993), part. II, p. 627 .

9 S. A. Fulling, SIAM J. Math. Anal. 14, 780 (1983).

10 T. A. Osborn and F. H. Molzahn, Phys. Rev. A 34, 1696 (1986).

11 F. H. Molzahn, T. A. Osborn and S. A. Fulling, Ann. Phys. (N.Y.) 204, 64 (1990); Ann. Phys. (N.Y.) 214, 102 (1992). 
12 F. H. Molzahn and T. A. Osborn, Ann. Phys. (N. Y.) 230, 343 (1994).

13 I. G. Avramidi and R. Schimming, The heat kernel coefficients to the matrix Schrödinger operator, University of Greifswald (1995), hep-th/9501026.

14 R. Camporesi, Phys. Rep. 196, 1 (1990).

15 E. Elizalde, S. D. Odintsov, A. Romeo, A. A. Bytsenko and S. Zerbini, Zeta regularization techniques with applications (World Scientific, Singapore, 1994).

16 I. G. Avramidi, Phys. Lett. B 236, 443 (1990); Yad. Fiz. 49 (1989) 1185.

17 A. O. Barvinsky and G. A. Vilkovisky, Nucl. Phys. B 282, 163 (1987); Nucl. Phys. B 333, 471 (1990).

18 G. A. Vilkovisky, in Publication de l' Institut de Recherche Mathématique Avancée, (R. C. P. 43 Strasbourg, 1992), p. 203.

19 A. O. Barvinsky, Yu. V. Gusev, G. A. Vilkovisky and V. V. Zhytnikov, J. Math. Phys. 35, 3525 (1994); Covariant perturbation theory (IY), University of Manitoba (1993).

20 L. Parker and D. J. Toms, Phys. Rev. D 31, 953 (1985).

21 I. Jack and L. Parker, Phys. Rev. D 31, 2439 (1985).

22 J. A. Zuk, Phys. Rev. D 34, 1791 (1986); Phys. Rev. D 33, 3645 (1986).

23 I. G. Avramidi, Phys. Lett. B 305, 27 (1993).

24 I. G. Avramidi, Covariant methods for calculating the low-energy effective action in quantum field theory and quantum gravity, University of Greifswald (1994), grqc/9403036.

25 I. G. Avramidi, New algebraic methods for calculating the heat kernel and the effective action in quantum gravity and gauge theories, University of Greifswald (1994), gr-qc/9408028; in Heat Kernel Techniques and Quantum Gravity, Discourses in Mathematics and Its Applications, No. 4, edited by S. A. Fulling, Texas A\&M University, (College Station, Texas, 1995), to appear.

26 I. G. Avramidi, Phys. Lett. B 336, 171 (1994).

27 I. G. Avramidi, A new algebraic approach for calculating the heat kernel in quantum gravity, University of Greifswald (1994), hep-th/9406047, submitted to J. Math. Phys.

28 I. G. Avramidi, Covariant algebraic calculation of the one-loop effective potential in 
non-Abelian gauge theory and a new approach to stability problem, University of Greifswald (1994), gr-qc/9403035, J. Math. Phys. (1995), to appear.

29 A. Erdelyi, W. Magnus, F. Oberhettinger and F. G. Tricomi, Higher Transcendental Functions, vol. I, (McGraw-Hill, New York, 1953). 